EPJ Web of Conferences 53, 08010 (2013)

DOI: $10.1051 /$ epjconf/20135308010

(C) Owned by the authors, published by EDP Sciences, 2013

\title{
Observation of microwave emission from extensive air showers with CROME
}

\author{
R. Šmída ${ }^{1, a}$, S. Baur ${ }^{1}$, M. Bertaina ${ }^{5}$, J. Blümer ${ }^{1}$, A. Chiavassa ${ }^{5}$, R. Engel ${ }^{1}$, \\ A. Haungs ${ }^{1}$, T. Huege ${ }^{1}$, K.-H. Kampert ${ }^{3}$, H. Klages ${ }^{1}$, M. Kleifges ${ }^{1}$, O. Krömer ${ }^{1}$, \\ M. Ludwig ${ }^{1}$, S. Mathys ${ }^{3}$, P. Neunteufel ${ }^{1}$, J. Pekala ${ }^{4}$, J. Rautenberg ${ }^{3}$, \\ M. Riegel ${ }^{1}$, M. Roth ${ }^{1}$, F. Salamida ${ }^{2}$, H. Schieler ${ }^{1}$, J. Stasielak ${ }^{4}$, M. Unger ${ }^{1}$, \\ M. Weber ${ }^{1}$, F. Werner ${ }^{1}$, H. Wilczyński ${ }^{4}$ and J. Wochele ${ }^{1}$ \\ ${ }^{1}$ Karlsruhe Institute of Technology (KIT), Karlsruhe, Germany \\ 2 Università dell'Aquila and INFN, L'Aquila, Italy \\ ${ }^{3}$ Bergische Universität Wuppertal, Wuppertal, Germany \\ ${ }^{4}$ Institute of Nuclear Physics PAN, Krakow, Poland \\ ${ }^{5}$ Università di Torino and Sezione INFN, Torino, Italy
}

\begin{abstract}
We report on the measurement of microwave radio signals from air showers with the CROME (Cosmic Ray Observation via Microwave Emission) experiment. CROME is located in the center of the KASCADE-Grande air shower array. The radio signals of the CROME antennas are stored for each highenergy trigger from the KASCADE-Grande array and matched offline with the KASCADE-Grande data. After almost one year of data taking microwave signals have been observed for more than ten air showers.
\end{abstract}

\section{INTRODUCTION}

The goal of CROME is the measurement of microwave signals expected to be emitted by extensive air showers. In two test-beam measurements, Gorham et al. [1] observed an isotropic emission by very low energy electrons, suggesting a measurable signal for air shower plasmas. The predicted signal would allow, in the best case, a calorimetric measurement of the longitudinal development of extensive air showers with almost 100\% duty cycle. Such a promising scenario has motivated several groups to build microwave experiments from commercially available components.

The two frequency bands allocated to downlink satellite communication, the extended $\mathrm{C}$ band $(3.4-4.2 \mathrm{GHz})$ and $\mathrm{Ku}$ band $(10.7-12.7 \mathrm{GHz})$, are particularly well-suited for an observation of air shower signals as they are characterized by

- very low natural background radiation,

- very little human-made radio frequency interference (RFI) [2], and

- negligible absorption in the atmosphere.

Moreover, commercial low-noise receivers for the $\mathrm{C}$ and $\mathrm{Ku}$ bands are commonly available in the form of low-noise block downconverters (LNBs).

There are several experiments searching for a shower-like trigger pattern in the atmospheric microwave radiation. These are the pioneering AMBER (Air-shower Microwave Bremsstrahlung Radiometer) [1] detector and various other setups including MIDAS (Microwave Detection of

ae-mail: radomir.smida@kit.edu

This is an Open Access article distributed under the terms of the Creative Commons Attribution License 2.0, which permits unrestricted use, distribution, and reproduction in any medium, provided the original work is properly cited. 


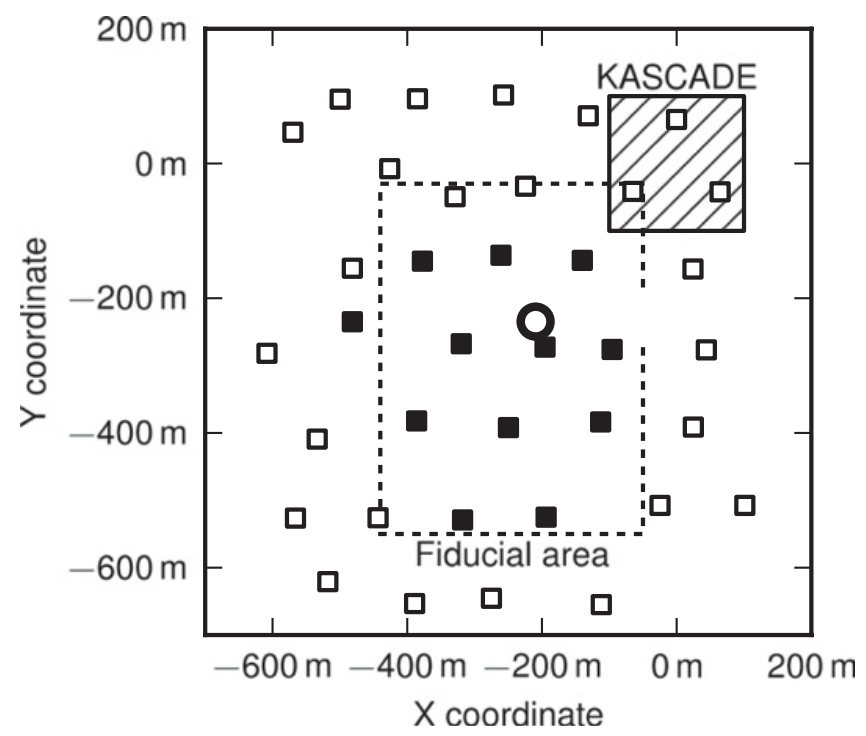

Figure 1. Position of the CROME microwave experiment in the KASCADE-Grande array. Full squares indicate the scintillator stations that provide the trigger for CROME. The information from the smaller but much denser KASCADE array (upper right corner) with separate electron and muon counters is used for estimating the number of muons in the showers.

Air Showers) [3], EASIER (Extensive Air Shower Identification using Electron Radiometer) [4], CROME [5] and two experiments in Japan [6, 7].

First unambiguous detections of microwave signals emitted by extensive air showers were presented at this conference. A clear signal was found with the EASIER setup [8] and the CROME experiment. The results of CROME, based on data taken between May 2011 and January 2012, are described in this proceeding contribution.

\section{THE CROME EXPERIMENT}

The CROME experiment is located inside the KASCADE-Grande cosmic-ray array [9] at the Karlsruhe Institute of Technology (KIT), see figure 1. It consists of various radio antennas covering a wide range of frequency. The readout of the antennas is triggered by KASCADE-Grande, which is optimized to detect air showers in the range from $10^{15.5}$ to $10^{18} \mathrm{eV}$. The trigger signal for CROME is built on a coincident measurement of the 12 inner KASCADE-Grande stations.

The leading CROME instruments are $\mathrm{C}$ band antennas. The $\mathrm{C}$ band detectors are parabolic reflectors of $335 \mathrm{~cm}$ diameter and a focal length of $119 \mathrm{~cm}$. The antennas have a gain of about $40 \mathrm{dBi}$ and are pointed vertically upward to minimize the distance to the shower maximum and to profit from the corresponding time compression of a possible shower signal.

The cameras in all $\mathrm{C}$ band antennas are equipped with 9 linearly polarized $\mathrm{C}$ band receivers (reflector-matched feedhorns and low-noise LNBs). The radiation pattern of the central and two offcenter receivers is shown in figure 2 . The data were measured with a calibrated airborne transmitter and are consistent with simulations for the far field zone obtained with the software package GRASP [11]. The main lobes are clearly visible and the half-power beam width is about $1.6^{\circ}$ for all channels. Aberration effects can be seen for off-center receivers. 


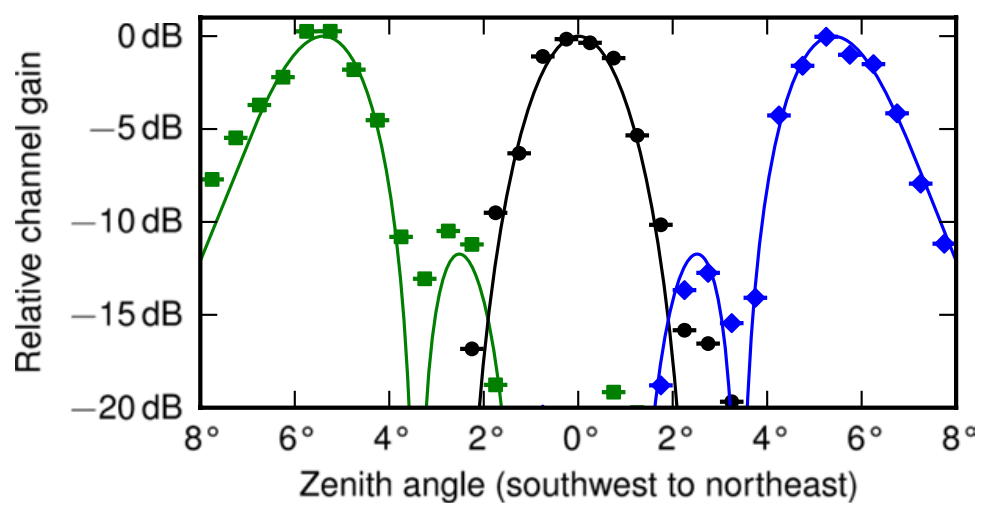

Figure 2. Two-dimensional radiation pattern of the central and two off-center receivers measured by the airborne radio source. Points indicate measured values and curves show simulated far field radiation patterns. The gain pattern has been normalized separately for each feed.

The polarization of a receiver is defined by the orientation of the feedhorn in a camera and the camera orientation towards the magnetic north. In each camera, there are two groups of receivers, five with approximate North-South (NS) and four with almost East-West (EW) orientation.

An analog DAQ chain of one receiver channel starts with the LNB (Norsat 8215F) which transforms an incoming signal from a frequency band of $3.4-4.2 \mathrm{GHz}$ down to $0.95-1.75 \mathrm{GHz}$. The amplifier in the LNB is powered by a bias tee (Mini-Circuits ZFBT-352-FT+). An attenuator of $6 \mathrm{~dB}$ (Radiall $\mathrm{R} 411806$ 124) is used to match the signal strength with the DAQ electronics and to suppress reflections due to an impedance mismatching between the LNB (75X) and the power detector (50X). Another component in the chain is a high pass filter (Mini-Circuits-VHF-1200) which reduces the bandwidth to $570 \mathrm{MHz}$ and suppresses RFI from airplane altimeter radars.

A logarithmic power detector (Analog Devices AD 8318 in Mini-Circuits-ZX47-60+) is used to measure the envelope of the antenna signals. The expected duration of the radio pulses is very short $(\sim 10 \mathrm{~ns})$ for a vertical air shower, requiring fast DAQ electronics. By removing an external capacitor in the power detector, the response time of the whole chain of electronics together with the LNB was significantly reduced to $\sim 9$ ns for 10 -to- $90 \%$ rise time (corresponding to an exponential time constant of $\sim 4 \mathrm{~ns})$.

The signal is sampled by 4-channel digitizers (PicoScope 6402 and 6403) with a sampling time of $0.8 \mathrm{~ns}$ and 8-bit dynamic range for $\pm 50 \mathrm{mV}$. All channels are read out in a time window of $10 \mu \mathrm{s}$ before and after the trigger delivered by KASCADE-Grande.

A GPS clock identical to the one of KASCADE-Grande is used to assign a time stamp to the triggers to merge the CROME and KASCADE-Grande data later. In addition, the arrival time of the shower front relative to the KASCADE-Grande trigger signal is determined by directly digitizing the discriminator signal of one station of KASCADE-Grande.

The reconstructed arrival direction, core position, and energy of the showers recorded by the KASCADE-Grande array are used in the further analysis. The Grande reconstruction accuracy is $\sim 0.8^{\circ}$ for the arrival direction, $\sim 6 \mathrm{~m}$ for the core position and about $20 \%$ for the energy reconstruction if the standard quality cuts are applied [9].

In addition to the $\mathrm{C}$ band antennas, an antenna for the $\mathrm{L}$ band $(1-2 \mathrm{GHz})$ is used to search for signals of lower frequency. Unfortunately, this frequency range is heavily affected by RFI [2], which makes the measurement of weak signals very challenging. Moreover, the CROME setup was extended by log periodic dipole antennas (LPDAs) that were previously used in the LOPES-Star experiment [10]. The LPDAs and the corresponding custom-made electronics are designed to detect radio signals between 38 and $80 \mathrm{MHz}$. 

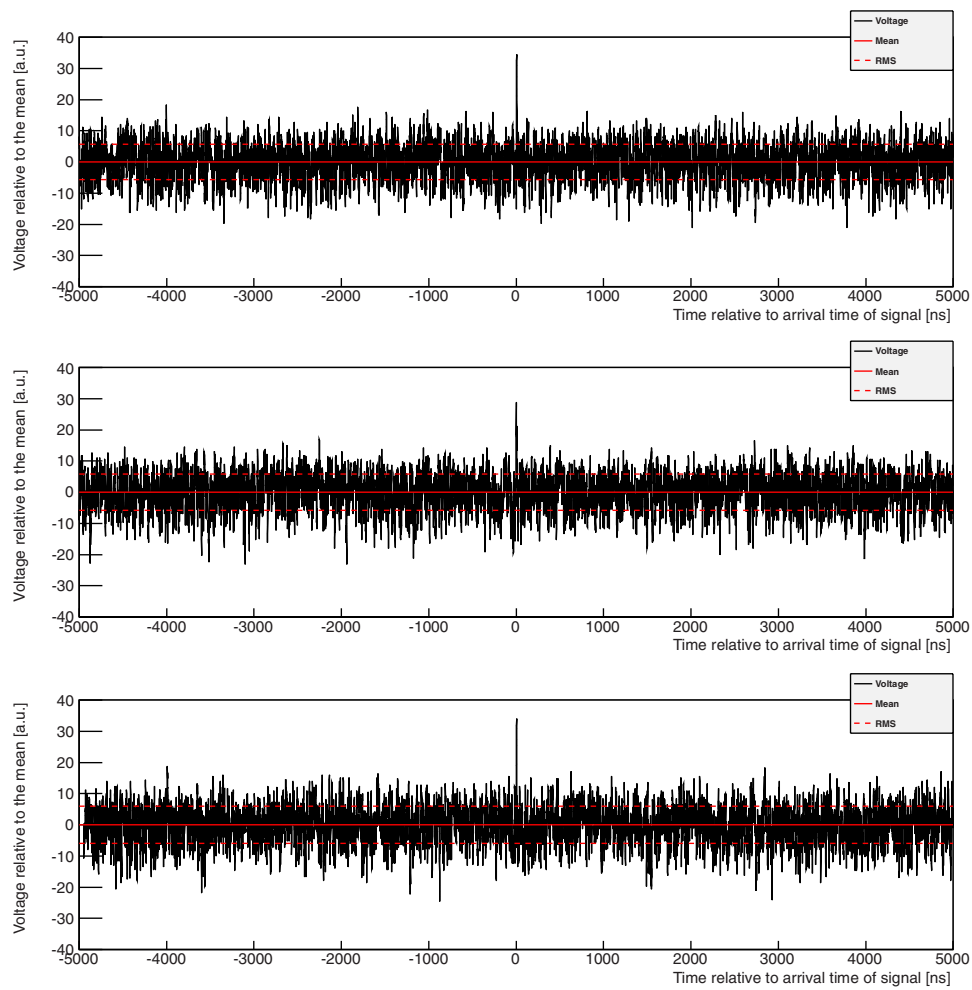

Figure 3. Traces for the three events with the highest signal. Voltage measured $5 \mu$ s before and after the rise of shower signal is plotted relative to the mean. The dashed red lines show the RMS calculated for the whole trace.

\section{CHARACTERISTICS OF AIR SHOWERS WITH MICROWAVE SIGNAL}

In the following we discuss those events for which the deviation of the measured voltage (which has logarithmic scale) within a $100 \mathrm{~ns}$ window (i.e. 125 samples) around the expected arrival time is more than $3 \sigma$. The found correlation between the field of view of the receivers and the shower geometry is expected to increase the significance of the event candidates, but has not been used in this estimate.

Twelve events with microwave signals arriving in time coincidence with air showers were detected between May 2011 and January 2012. All measured signals had a few nanoseconds duration.

The $\mathrm{C}$ band traces of the three events with the highest signal are shown in figure 3 as voltage relative to the mean voltage. The time window is centered at the signal peak. The arrival time of the microwave signal is approximately $5 \mu$ s before the KASCADE-Grande trigger for the events, which is in very good agreement with the calculated propagation time and measured delay of the trigger signal. The signal peak is clearly visible in the zoomed traces (see Fig. 4).

It should be noted that a shower signal was typically found in only one channel. This excludes most RFI from sources located outside (e.g. airplanes, ANKA synchrotron source, any broadband emitter) or inside the DAQ container where the readout electronics is located. Also, the receivers detecting microwave pulses differ from event to event and from antenna to antenna.

All twelve measured signals have common features, which can be summarized as follows:

- The duration of the observed signal is about $10 \mathrm{~ns}$.

- The energy threshold for a detection of the events is about $10^{16.5} \mathrm{eV}$. 

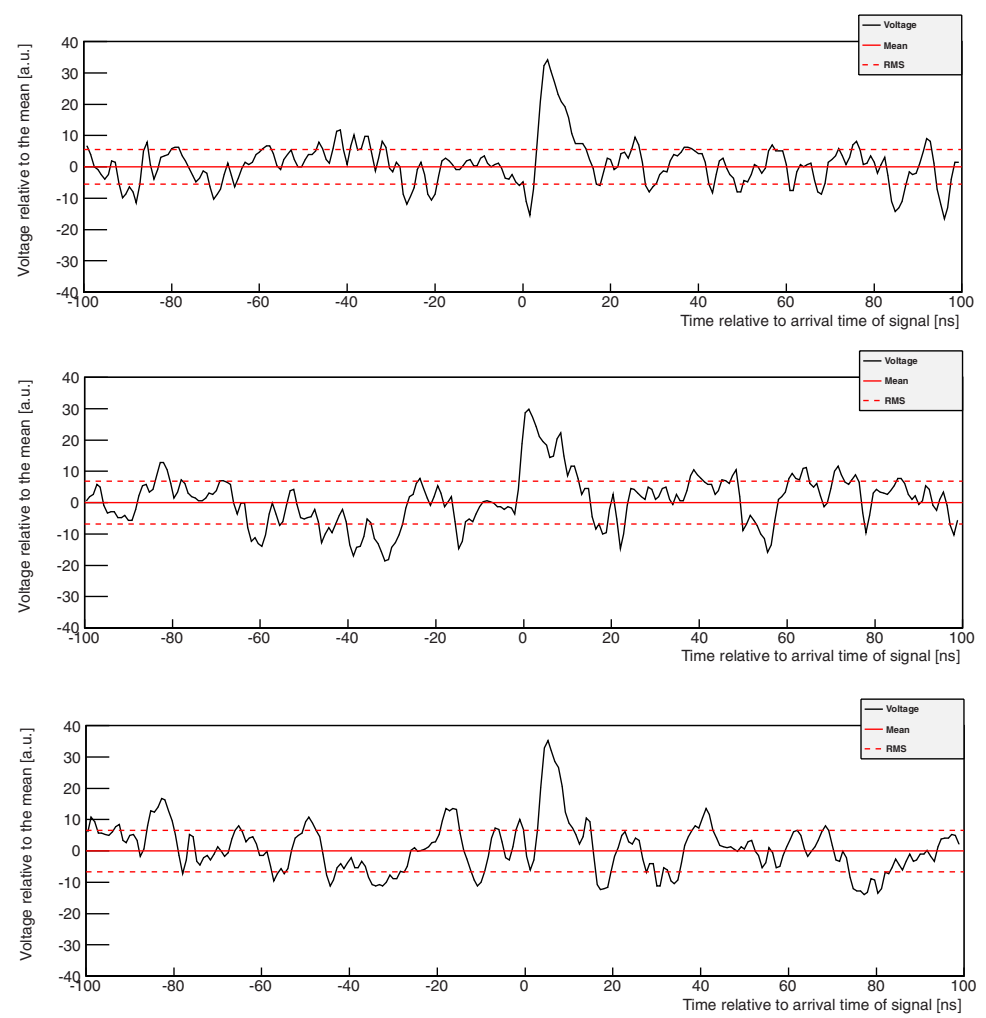

Figure 4. Same as in Figure 3, but the signal is zoomed to $100 \mathrm{~ns}$ before and after the rise of shower signal.

- The angle between the reconstructed shower axis and the optical axis of a receiver (boresight direction), is very small for all showers. The same applies if one calculates the angle between the line of sight to the shower disc at $4 \mathrm{~km}$ altitude and the boresight direction of the receivers with detected signal.

- The distances between the core positions of the showers and the antennas are in the range of $80 \mathrm{~m}$ to $150 \mathrm{~m}$.

- Events have been detected with receivers of different polarization angles relative to the geomagnetic East-West direction. No particular polarization direction is favored within the limited statistics available so far.

A clear VHF radio signal was measured for the most energetic showers. Not surprisingly, a stronger radio signal is measured in the EW polarization direction. A systematic analysis of the VHF data and possible correlations with the $\mathrm{GHz}$ traces is in preparation.

\section{DISCUSSION OF EMISSION MECHANISMS}

Possible sources of radio emission include

- coherent radiation due to the time-varying charge excess in an air shower,

- incoherent radiation of individual, charged shower particles as observed, for example, with imaging atmospheric Cherenkov telescopes,

- coherent radiation due to the deflection of electrons and positrons in the geomagnetic field, creating time-varying transverse currents, 
- molecular bremsstrahlung, and

- radio waves from external sources that are reflected off the shower plasma (see e.g. [12]).

In general, the observed emission pattern shows features typical for Cherenkov radiation. However, due to the limited statistics and the detection threshold of the CROME antennas it cannot be excluded that a Cherenkov-like compression of the signal is needed to reach the detection threshold. In this case a strong bias of the selected events in favor of the Cherenkov angle is expected.

In all cases, the signal emitted (or reflected) by the source will be compressed in time to a few ns duration, or conversely to very high frequency. Depending on the viewing angle to the shower axis, a reflected signal appearing in the $\mathrm{MHz}$ range at large shower angles can be compressed to $\mathrm{GHz}$ frequencies close to the Cherenkov angle.

At the current stage of the analysis of the events it is not possible to distinguish between coherent Cherenkov radiation (Askaryan effect), incoherent Cherenkov radiation by individual charged shower particles, or the reflection of radio waves from external sources as the dominant process that leads to the observed microwave emission. Also, molecular bremsstrahlung, if emitted predominantly in forward direction, is not ruled out.

\section{CONCLUSION}

Using the CROME experiment, we have observed microwave emission of high-energy air showers. In total, 12 cosmic-ray events with an obvious microwave signal in the $\mathrm{C}$ band have been found in the data taken between May 2011 and January 2012. The showers with an reconstructed energy greater than $10^{17} \mathrm{eV}$ show also a strong signal in the VHF band.

The CROME data provide a unique opportunity for studying different models of radio emission from air showers. An important piece of information about the microwave emission process is the polarization. The data reported here does not show a preferred polarization direction within the measurement uncertainties and the limited statistics. The signal polarization will be measured more reliably with dual polarized receivers that are currently being installed. In addition, a statistically significant improvement of the shower core distribution relative to the antennas will be obtained during next months.

We are going to perform simulations for the observed cosmic ray showers for all three frequency bands. The simulated predictions for polarization, energy dependence of the signal strength, and core position will be compared with the measurements.

It is our pleasure to acknowledge the interaction and collaboration with many colleagues from the KASCADEGrande and Pierre Auger Collaboration. This work has been supported in part by the KIT start-up grant 2066995641, the ASPERA project BMBF 05A11VKA and 05A11PXA, the Helmholtz-University Young Investigators Group VH-NG-413 and the National Centre for Research and Development (NCBiR).

\section{References}

[1] P.W. Gorham et al., Physical Review D 78 (2008) 032007

[2] European Radiocommunications Committee, ERC report 25 (2009)

[3] J. Alvarez-Muniz, submitted to Physical Review Letters, (2012) arXiv:1205.5785

[4] P. Allison for the Pierre Auger Collaboration, Proc. of the 32nd Int. Cosmic Ray Conf. (Beijing) 2011, arXiv:1107.4807

[5] R. Šmída et al., Proc. of the 32nd Int. Cosmic Ray Conf. (Beijing) 2011, arXiv:1108.0588

[6] K. Kuramoto et al., Proc. of the 32nd Int. Cosmic Ray Conf. (Beijing) 2011

[7] T. Yamamoto et al., Proc. of the 32nd Int. Cosmic Ray Conf. (Beijing) 2011 


\section{UHECR 2012}

[8] P. Facal for the Pierre Auger Collaboration, Proc. of the Int. Symposium on Future Directions in UHECR Physics (CERN) 2012

[9] W.D. Apel et al., Nuclear Instruments and Methods in Physics Research A 620 (2010) 202-216

[10] W.D. Apel et al., Astroparticle Physics 32 (2010) 294-303

[11] http://www.ticra.com/

[12] J. Stasielak et al., Proc. of the Int. Symposium on Future Directions in UHECR Physics (CERN) 2012 